\title{
Systematically and Fast Processing Fault on Power Distribution Grids Based on a Simplified Model
}

\section{Part I: Basic Approaches}

\author{
Liang $\mathrm{Ma}^{1}$, Yi Xun ${ }^{1}$, Zhihua Zhang ${ }^{2, *}$, Xiaoqing Zhang ${ }^{2}$ and Di Yao ${ }^{3}$ \\ ${ }^{1}$ Xi' an Power Supply Company, State Grid Shaanxi Electric Power Company, Xi'an 710050, China \\ ${ }^{2}$ Electric Power Research Institute, State Grid Shaanxi Electric Power Company, Xi' an 710032, China \\ ${ }^{3}$ Department of Electronic and Information, Xi' an Polytechnic University, Xi' an 710048, China \\ ${ }^{*}$ Corresponding author
}

\begin{abstract}
This is Part I of a two-part paper which presents a methodology for fault location and isolation and service restoration for power distribution grids. Concepts of node, region, connected sub-grid, and simplified model of distribution grids are introduced. A simplified load flow analysis is discussed based on node-and-region load transformations. Complicated circuit analysis is avoided. Five criteria are proposed for fault location, including the distinguishability of multiple single-region faults and multiple multi-region faults. The fault is first located in a monitoring region and then in an action region. After that the action nodes of the action region are tripped to isolate the fault. Two service restoration schemes among a total of four are introduced in Part I. Tabu search is applied to seek for a close-tooptimal service restoration solution. The proposed approaches can be used in a grid of any scale. They have been imbedded in $\mathbf{2 6}$ power distribution automation systems of $10 \mathrm{kV}$ grids since 2013 , and have been proven to be a great success.
\end{abstract}

Keywords-distribution networks; fault location; load flow analysis; power distribution faults; power system restoration

\section{INTRODUCTION}

More than eighty-five percent of outages in power distribution grids are caused by various faults [1]. Therefore, fast and effective fault location, isolation, and service restoration (FLISR) is of great importance to improve the service reliability.

The implementation of FLISR is based on local intelligence, distributed intelligence [2], or centralized intelligence [1].

Fault types fall into the following five categories: singlephase-ground, two-phase, two-phase-ground (or phase-phaseground), three-phase, and three-phase-ground. Except the single-phase-ground fault, the other 4 types are interphase faults which generally produce a large fault current.

A large amount of literature has been published on fault location methods. There are a number of properties along which the methods are classified.

Based on the states of the fault information used, fault location methods can be divided into two types. Steady-state methods utilize steady-state voltages and/or currents or the fundamental frequency components extracted from the transient signals [3].Transient methods utilize the faultoriginated electromagnetic transients of voltages and/or currents. The most common steady-state methods are based on impedance, while some transient methods are based on traveling waves [4], [5]. Impedance-based methods and traveling-wave methods are outlined in an IEEE standard [6].

Based on the accuracy, fault location methods can be divided into two types, namely pinpointing the fault [7]-[10] and locating the faulted region [11]-[13]. The former is the topic of most of the related works of the literature, and is preferred by the patrol men. Synchronized voltage and current phasors are involved for a higher accuracy [14]-[21]. The latter has been discussed far less than the former. But it needs information with less accuracy than the former, and its related algorithms are much faster and more practical. Therefore, it is preferred in power distribution automation systems. The approaches proposed in this paper belong to the second type.

Based on the mathematical methods used, fault location methods can be divided into two types. Type one locates the fault by analytically solving equation sets. Whereas type two does it in logical ways whereby quantities are converted into "1"s or "0"s [12], [22], [23].

Based on the sources of the information used, fault location methods can be divided into the passive type and the active type. The passive type uses fault information which is generated by the power sources inside the grid, and is more popular than the active type. Whereas the active type uses signals injected into the grid by outside equipment [24], which is particularly suitable for a de-energized faulted feeder [25].

With all the fault location methods, it is a challenge to distinguish the most suitable one for a certain grid. An approach is proposed to rank the available methods in [17].

Anyway, few works of literature have dealt with the whole process of FLISR. This paper presents a methodology for FLISR on power distribution grids which are equipped with centralized automation systems. A distinguishing feature of the proposed methodology is that it is based on a simplified grid model. Load flow calculation, fault location, and network reconfiguration are carried out within a connected sub-grid which is made up of regions and which is of a far much smaller 
scale compared with the whole grid. The load flow is calculated through basic arithmetic operations rather than conventional complicated circuit analysis. The faulted region is located by checking the directions of the fault current or power at each region. The proposed methodology can be used in a grid of any scale, however large it is. Tabu search is applied to seek for a close-to-optimal service restoration solution. The algorithm for the methodology has been imbedded in the master stations of 26 distribution automation systems. After a fault has been detected, the whole procedure of FLISR is carried out automatically in sequence within a quite short time if the switches are remotely controlled.

This paper consists of two parts. Part I, i.e. this part, deals with basic concepts and approaches for FLISR. Part II will deal with advanced approaches.

\section{SIMPLIFIED MODEL OF DISTRIBUTION GRIDS}

Based on the graph theory, a simplified model of distribution grids is established for FLISR.

\section{A. Reference Direction}

Due to the introduction of distribution generations (DG) into power distribution grids, the power flow through a grid becomes bidirectional. Nonetheless, we still define the direction from the main power source to the terminals of a feeder as the reference direction. Thus, the terms upstream and downstream have the same meanings as they do in a grids without DGs.

\section{B. Node}

A point on the feeder where a switch or fault monitoring device is installed is called a node. This definition is completely different from the traditional one. Nodes are classified into four types as follows.

Action nodes are the switches which are used to connect or disconnect the branches. Action nodes can be divided into two sub-types, namely the sectionalizing action node based on the sectionalizer which is normally closed, and the tie action node based on the tie switch which is normally open.

Monitoring nodes are the nodes where fault indicators are installed. Usually an action node is also a monitoring node. But the inverse is not always true. For example, a node with a fault indicator but without any switch is only a monitoring node.

Ending nodes are the terminals of a trunk, a branch or a lateral. The state of an ending node is constantly open.

Source nodes are the main power sources. They are actually the circuit breakers in the substations, and hence are action nodes. The switches connecting the distribution generations (DG) to the main distribution grid can also be regarded as source nodes. Thus, source nodes can be classified into two groups: the main source node and the DG source node.

The four types of node are demonstrated in Figure I. The solid icons indicate closed states, while the hollow icons indicate open states. The parallelograms are main source nodes, representing the line circuit breakers in the substations. The solid circles are sectionalizing action nodes, and the hollow ones are tie action nodes. The squares are monitoring nodes, representing fault indicators. The triangles are ending nodes.

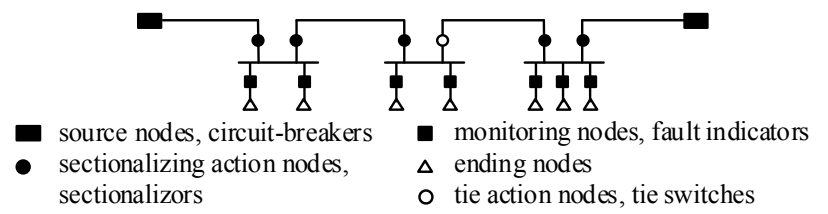

FIGURE I. EXAMPLE TO ILLUSTRATE THE FOUR TYPES OF NODE

\section{Region}

A region is a sub-grid surrounded by several nodes of the same type, and has no any node of the same type inside.

Regions can be classified into two types.

Action regions are the regions surrounded by action nodes or ending nodes without any action nodes inside. An action region is the smallest unit for fault isolation and load transfer.

Monitoring regions are the regions surrounded by monitoring nodes or ending nodes without any monitoring nodes inside. A monitoring region is the smallest unit for fault location.

Examples for different regions are illustrated in Figure II by dashed lines. It can be easily seen and understood that an action region contains one or more monitoring regions.

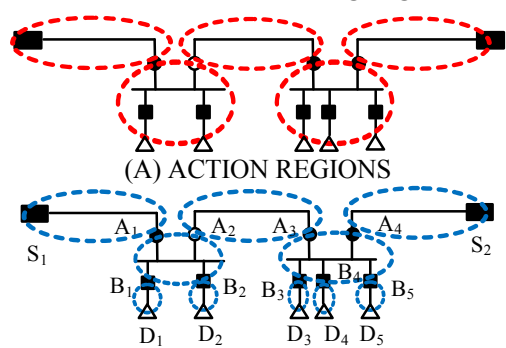

(B) MONITORING REGIONS

FIGURE II. TWO TYPES OF REGION

\section{Connected Sub-Grid}

A connected sub-grid is made up with a series of regions. It has features as follows:

-The regions are all connected when all of the tie switches in the connected sub-grid are closed.

-The connected sub-grid must not cover a substation bus. Therefore, load transfer can be limited within a connected sub-grid.

-The number of the regions reaches maximum.

A connected sub-grid is the smallest unit for grid reconfiguration and service restoration. No matter how large a distribution grid is, the service restoration can be carried out within a connected sub-grid, which is generally of a much smaller scale compared with the whole grid. Figure III illustrates three connected sub-grids by dashed lines. 


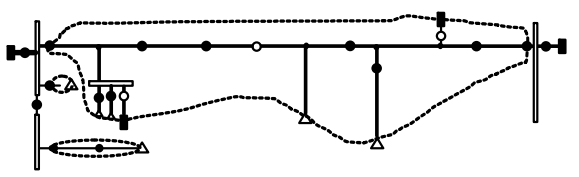

FIGURE III. CONNECTED SUB-GRIDS

\section{E. Node Hierarchy}

Node and region hierarchies are defined for single main source radial distribution grids. The hierarchy levels are limited within a single connected sub-grid. In other words, nodes or regions in different connected sub-grids have no relationship, though the connected sub-grids are in the same distribution grid.

The hierarchy level of the $\mathrm{i}$-th node is defined as $\mathrm{HN}(\mathrm{i})=\mathrm{N}$,

where $\mathrm{N}$ is the number of nodes from the main source node (excluded) of the feeder to the i-th node. It should be noted that $i$, usually the sequence number of a node, here is just a sign representing this node. However, $\mathrm{N}$ is strictly counted from the main source node. Several nodes can have the same hierarchy level $\mathrm{N}$.

The hierarchy level of the main source node is zero.

If $\mathrm{HN}(\mathrm{j})-\mathrm{HN}(\mathrm{i})>0$, the $\mathrm{i}$-th node is a senior node to the $\mathrm{j}$-th node, and the $\mathrm{j}$-th node a junior node to the $\mathrm{i}$-th node.

If the $i$-th and $j$-th nodes are adjacent, and $\mathrm{HN}(\mathrm{j})-\mathrm{HN}(\mathrm{i})=1$, the $i$-th node is the father node of the $j$-th node, and the $j$-th node is the son node of the $\mathrm{i}$-th node.

In the example in Figure IV, the numbers in brackets indicate the hierarchy levels of the nodes, and the numbers beside the nodes are the sequence numbers of the nodes. The hierarchy levels are visualized by dashed curve lines. Some of them are $\mathrm{HN}(12)=1, \mathrm{HN}(11)=2, \quad \mathrm{HN}(7)=\mathrm{HN}(8)=\mathrm{HN}(9)=$ $\mathrm{HN}(10)=3$. Obviously, node 11 is the father node of nodes 7,8 , 9 , and 10 .

The node hierarchy is the foundation of the region hierarchy.

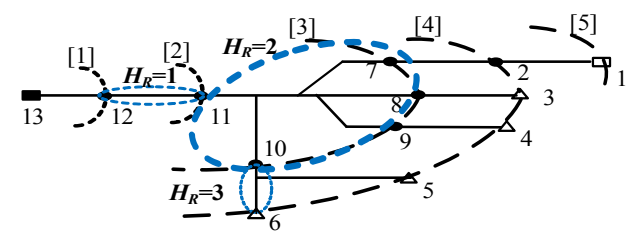

FIGURE IV. EXAMPLE OF NODE AND REGION HIERARCHIES

\section{F. Region Hierarchy}

The nodes of a region are called the terminals of the region. The terminal with the smallest hierarchy level is the enter terminal of the region. While the other terminals are the out terminals of the region. A region can be expressed as Re (enter terminal, out terminal 1 , out terminal $2, \ldots$ ).

The hierarchy level of the $i$-th region, denoted by $H_{R}(i)$ or $H_{R}$ (enter terminal, out terminal 1 , out terminal $2, \ldots$ ), is equal to the hierarchy level of its enter terminal.
Some hierarchy levels in Figure $\mathrm{II}(\mathrm{B})$ are $H_{R}(A 1, A 2, B 1$, B2) $=1, H_{R}(B 1, D 1)=2$.

The terms of father, son, senior, and junior for region hierarchies are defined in a similar way as those for node hierarchies.

If $H_{R}(i)<H_{R}(j)$, and there is a relationship that the $j$-th region is the son of son of ... of $i$-th region, the two regions have an immediate family relationship. The $i$-th region is a direct ancestor of the $j$-th region, and the $j$-th region is a direct descendant of the $i$-th region. This immediate family relationship can be interpreted in another way. If a fault occurs in the $j$-th region, the fault current flows through not only the enter terminal but also one of the out terminals of the $i$-th region. This interpretation is useful for distinguishing multiple faults, which will be discussed in the later text.

\section{G. Simplified Load Flow Analysis}

In this subsection, a simplified method of load flow analysis for distribution grids is described. The method is approximate but quite simple and fast, and therefore is suitable for service restoration.

1) Node Load and Region Load: The load flowing through the $i$-th node, denoted by $l_{N}(i)$, is named as the node load of this node. The load consumed within the $m$-th region, $l_{R}(m)$, is named as the region load of this region. $l_{R}(m)$ is also represented by $l_{R}(i, j, \ldots, k)$, where $i, j, \ldots$, and $k$ are the terminals.

The node load and region load can be expressed either in power or in current.

Sometimes, for instance, during service restoration, load rate is more convenient than the real load value. The load rate of the i-th node is defined as

$$
l_{N, \%}(i)=\frac{\left|l_{N}(i)\right|}{\left|l_{N, \max }(i)\right|}
$$

where $l_{N, \max }(i)$ is the capacity limit of the $i$-th node, and $l_{N, \%}(i)$ is the load rate of this node. Both the numerator and the denominator are absolute values.

When region loads are discussed later in the paper, they usually for action regions unless stated otherwise.

2) Node-to-Region Load Transformation: Region load $l_{R}(i$, $j, \ldots, k)$ is derived from node loads as

$$
l_{R}(i, j, \ldots, k)=l_{N}(i)-\sum_{\lambda \in \Lambda} l_{N}(\lambda)
$$

where $\Lambda$ is the set of the out terminals of the region, namely $\Lambda=\{j, \ldots, k\}$.

During the normal operation loads are obtained in real time. Region loads are calculated with ease by using (2), and then stored. Region loads will be essential for grid reconfiguration and service restoration after a fault. 
3) Region-to-Node Load Transformation: For node i, it is clear that

$$
l_{N}(i)=\sum_{\theta \in \Theta} l_{R}(\theta)
$$

where $\theta$ is the set of the $i$-th node's downstream regions.

From $(2), l_{N}(i)$ can also be calculated by

$$
l_{N}(i)=l_{R}(i, j, \ldots, k)+\sum_{\lambda \in \Lambda} l_{N}(\lambda)
$$

During grid reconfiguration and service restoration after a fault, node loads are quickly calculated by the region-to-node load transformation for a candidate scheme for service restoration.

The aforementioned method of node-to-region and regionto-node load transformations is the two steps of a simplified load flow calculation which is restricted within a connected sub-grid. This simplification significantly reduces computing load and the fault processing time.

4) An Example for Load Transformation: The distribution grid shown in Figure V is an example to illustrate the approach of the simplified load flow analysis. The numbers besides the nodes in Figure V(A) indicate the node loads in A. By conducting the node-to-region load transformation, the region loads are obtained and shown in brackets.

The grid is reconfigured as shown in Figure V(B). The new node loads achieved by region-to-node load transformation are shown in italic.

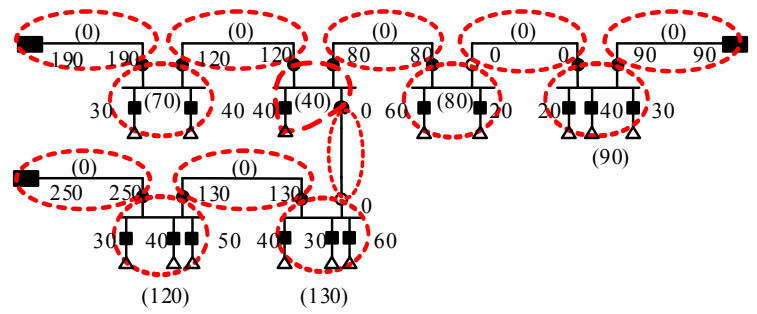

(A) LOADS BEFORE TRANSFORMATION

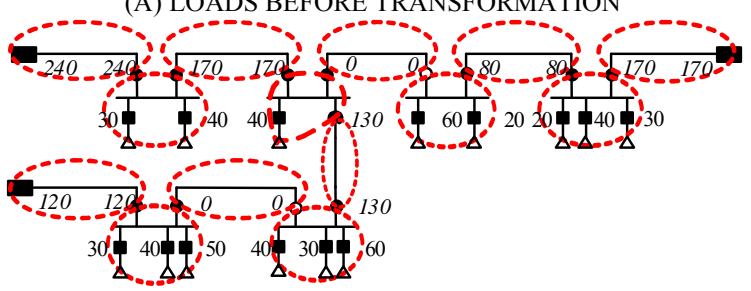

(B) NEW NODE LOADS AFTER RECONFIGURATION AND LOAD TRANSFORMATIONS

FIGURE V. AN EXAMPLE OF LOAD TRANSFORMATIONS

\section{LOCATION OF FAULTS WITH A LARGE FAULT CURRENT}

The criteria for fault location described in this section are based on sufficient information, and suitable for faults with a large fault current, namely inter-phase faults or single-phaseground faults on neutral effectively grounded grids. For faults on neutral non-effectively grounded grids, and for cases with no sufficient information (information missing and erroneous), the criteria will be discussed separately.

\section{A. Fault Location for Radial Grids - Criterion 1}

For a radial distribution grid, once a fault occurs on a feeder section, the fault current flows through all upstream monitoring nodes but none of the downstream monitoring nodes.

Thus, Criterion 1 is as follows:

For a region in a radial distribution grid, if its enter terminal endures the fault current and none of its out terminals endures the fault current, the fault occurs in the region. Otherwise, the region is healthy. In other words, the fault is in the region with the fault current flowing through just one terminal. An example is shown in Figure VI where a fault occurs in $\operatorname{Re}(4,3,5)$. The arrows beside the nodes indicate the direction of the fault current.

\section{B. Fault Location for Closed-Loop Grids-Criterion 2}

A radial distribution grid has only one main source. For a closed-loop distribution grid with multiple main sources, once a fault occurs, the fault current flows through all the nodes on the trunk and also the nodes on the branches that lead to the faulted section. Criterion 1 is not applicable anymore, because sometimes more than one terminals of the faulted region endures the fault current.

Criterion 2 is as follows:

For a region in a closed-loop distribution grid, if at least one terminal endures a fault current with the fault power flowing into the region, and none of the terminals endures a fault current with the fault power flowing out of the region, the fault occurs in the region. Otherwise, the region is healthy. An example is shown in Figure VII.

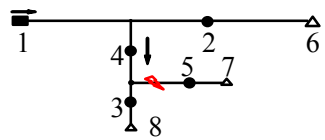

FIGURE VI. EXAMPLE FOR FAULT LOCATION CRITERION 1

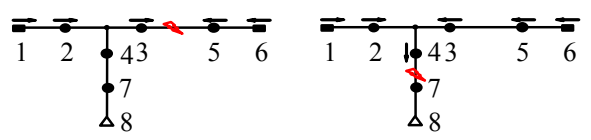

(A)

(B)

FIGURE VII. EXAMPLE FOR FAULT LOCATION CRITERION 2. (A) A FAULT ON THE TRUNK (B) A FAULT ON A BRANCH

To meet the requirement of Criterion 2, the current flowing through the monitoring nodes must be over the threshold set in advance, and the direction of the fault power must be detected too.

\section{Location for a Two-Phase-Ground Fault-Criterion 3}

The interphase short-circuit fault discussed in the previous sections occurs within a single monitoring region, which is referred as a single-region fault. For a neutral non-effectively grounded system, when a single-phase-ground fault occurs in 
region $\mathrm{A}$, the zero-sequence current is too small to trig the relay But the voltages on the healthy phases increase, and the corresponding insulation may fail, which results in another single-phase-ground fault in region $\mathrm{A}$ or in a different region. If the latter is the case, a two-phase-ground fault is initiated with two grounding points being in different regions. This kind of fault is called a two-region fault. Sometimes even a multiregion fault happens.

For a two-phase-ground fault, either a single-region one or a two-region one, the zero-sequence voltage is distinct.

Thus, Criterion 3 is as follows:

If the zero-sequence voltage resulted from a fault is over a preset threshold, the fault is a two-phase-ground short-circuit. The fault, with either one or two grounding points, can be located according to Criterion 1 for a radial grid, or Criterion 2 for a closed-loop grid.

A two-region fault is shown in Figure VIII. The faulted regions can be easily identified as regions $\mathrm{R}(\mathrm{A}, \mathrm{B}, \mathrm{C})$ and $\mathrm{R}(\mathrm{K}$, M).

Criterion 3 requires that the three phase currents should be detected.

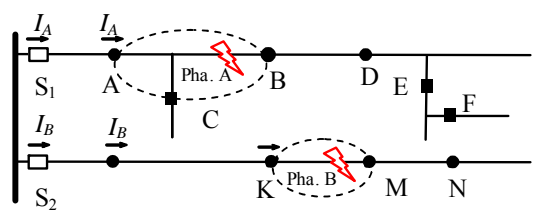

FIGURE VIII. EXAMPLE FOR A TWO-REGION TWO-PHASE-GROUND FAULT

\section{Distinguishability of Two Single-Region Faults-Criterion 4}

Due to the delay of transmission, the information of several faults which do not happen simultaneously may be received simultaneously by the master station of the distribution automation system. Therefore, these faults appear to have happened simultaneously. If the faults are coincident in one region, it is not necessary to distinguish them from the point of view of fault location. If they are not, a problem arises whether these faults can be distinguished. The problem is answered by Criterion 4.

\section{Criterion 4 is as follows:}

Two simultaneous single-region faults are distinguishable except that the two regions have an immediate family relationship.

Two single-region faults which have happened in different connected sub-grids simultaneously are distinguishable since they have no immediate family relationship.

Two examples are shown in Figure IX.

In Figure IX (A). Two faults occur in $\operatorname{Re}(\mathrm{A}, \mathrm{B}, \mathrm{C})$ and $\operatorname{Re}(\mathrm{E}$ F) simultaneously. The former is a direct ancestor region of the latter. Consequently, the two faults cannot be distinguished. In fact, according to Criterion 1 the fault is located only in $\operatorname{Re}(\mathrm{E}$, F). In Figure IX(B), two faults occur in $\operatorname{Re}(C, G)$ and $\operatorname{Re}(E, F)$ simultaneously. $\operatorname{Re}(\mathrm{C}, \mathrm{G})$ is a senior region to $\operatorname{Re}(\mathrm{E}, \mathrm{F})$, but they do not have an immediate family relationship. The two faults are distinguishable.

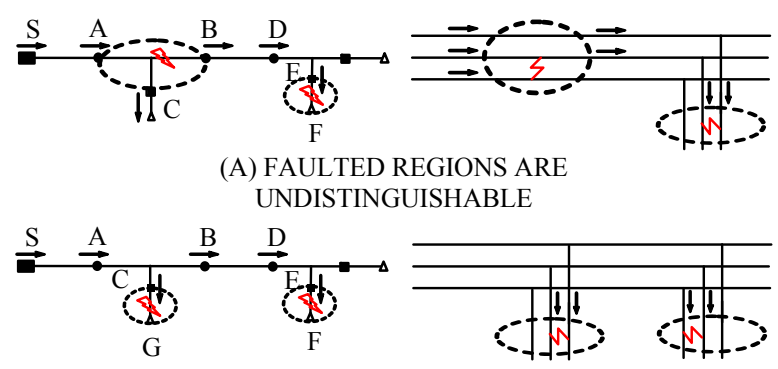

(B) FAULTED REGIONS ARE DISTINGUISHABLE

FIGURE IX. EXAMPLES TO ILLUSTRATE THE DISTINGUISHABILITY OF MULTIPLE SINGLE-REGION FAULTS

\section{E. Distinguishability of Multiple Multi-Region Faults- Criterion 5}

Criterion 5 is as follows:

For multiple multi-region faults, if faults happen on the same phase and the faulted regions have an immediate family relationship, the faults are undistinguishable from each other. Otherwise, they are distinguishable.

The usage of Criterion 5 is demonstrated in Figure X.

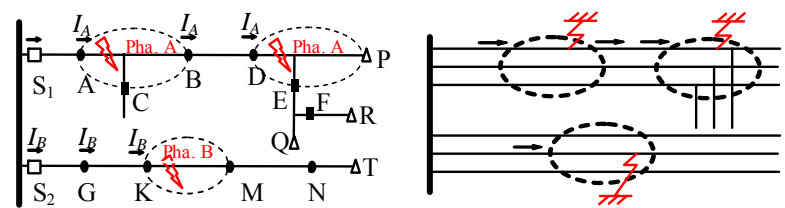

(A) TWO GROUNDING POINTS ON PHASE A AND ONE ON PHASE B

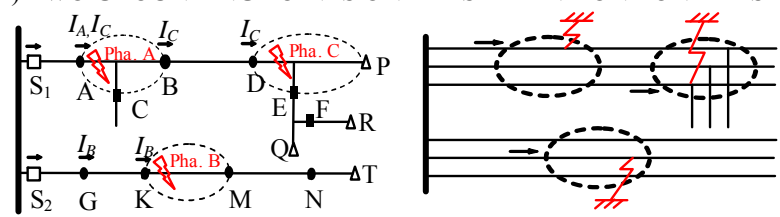

(B) ONE GROUNDING POINT ON EACH PHASE

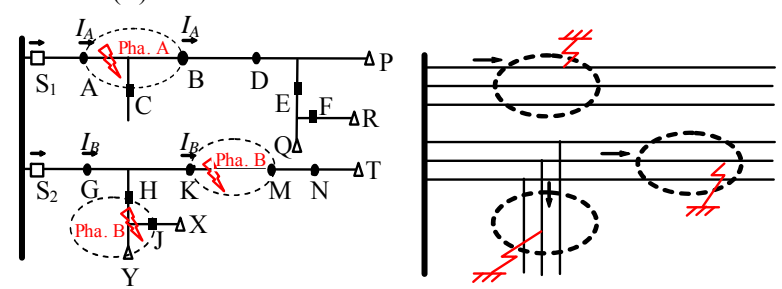

(C) ONE GROUNDING POINT ON PHASE A AND TWO ON PHASE B

FIGURE X. EXAMPLES TO DEMONSTRATE THE DISTINGUISHABILITY OF THE FAULTED REGIONS OF MULTIREGION FAULTS

In Figure $X(A)$, the two grounding points on phase $A$ are in two regions $\operatorname{Re}(\mathrm{A}, \mathrm{B}, \mathrm{C})$ and $\operatorname{Re}(\mathrm{D}, \mathrm{E}, \mathrm{P})$. The grounding point on phase $B$ is in $\operatorname{Re}(K, M) . \operatorname{Re}(A, B, C)$ and $\operatorname{Re}(D, E, P)$ have an immediate family relationship, and cannot be distinguished. However, $\operatorname{Re}(\mathrm{K}, \mathrm{M})$ is in another connected sub-grid. It has no immediate family relationship with $\operatorname{Re}(\mathrm{A}, \mathrm{B}, \mathrm{C})$ or $\operatorname{Re}(\mathrm{D}, \mathrm{E}, \mathrm{P})$, and can be identified as a faulted region. 
In Figure $\mathrm{X}(\mathrm{B})$, phases $\mathrm{A}, \mathrm{B}$, and $\mathrm{C}$ are grounded in different regions $\operatorname{Re}(\mathrm{A}, \mathrm{B}, \mathrm{C}), \operatorname{Re}(\mathrm{K}, \mathrm{M})$ and $\operatorname{Re}(\mathrm{D}, \mathrm{E}, \mathrm{P})$. Though $\operatorname{Re}(\mathrm{A}, \mathrm{B}, \mathrm{C})$ and $\operatorname{Re}(\mathrm{D}, \mathrm{E}, \mathrm{P})$ have an immediate family relationship, the faults are on two phases. Therefore, the two faulted regions can be distinguished. $\operatorname{Re}(\mathrm{K}, \mathrm{M})$ is in another connected sub-grid, and is identified as a faulted region.

In Figure $X(C)$, the grounding point on phase $A$ is in $\operatorname{Re}(A$, $\mathrm{B}, \mathrm{C})$, and the grounding points on phase $\mathrm{B}$ are in $\operatorname{Re}(\mathrm{K}, \mathrm{M})$ and $\operatorname{Re}(\mathrm{H}, \mathrm{Y}, \mathrm{J})$. The two faulted regions on phase $\mathrm{B}$ do not have an immediate family relationship, and are identified as two separate faulted regions. $\operatorname{Re}(\mathrm{A}, \mathrm{B}, \mathrm{C})$ is in another connected sub-grid, and is identified as a faulted region too.

\section{FAULT IsOLATION}

For a distribution automation system based on centralized intelligence, once the faulted region is located, the fault will be isolated by telecontrolled operations.

It has been pointed out in Section II that a monitoring region is the smallest unit for fault location, while an action region is the smallest unit for fault isolation and load transferring. An action region contains one or more monitoring regions. In other words, the fault isolation application will isolate an action region rather than the faulted region (the faulted region is a monitoring region).

The fault isolation process is as follows:

Step 1: Take a terminal of the faulted region.

Step 2: If the terminal is an action node or an ending node, put it into set $\Phi$.

Step 3: If the terminal is a monitoring node, search for all of its closest action nodes and/or ending nodes in the direction from the inside of the faulted region to the outside, put the findings into $\Phi$. If there is any monitoring node in the searching path, just ignore it.

Step 4: If all of the terminals are handled, go to Step 5. Otherwise, go to Step 1.

Step 5: Trip the action nodes in $\Phi$ by the function of remote control of the master station. Notably, some of the nodes have been tripped before by relay protection devices or other mechanisms, for example loss of voltage mechanism. Still some other nodes have to be kept open, such as tie switches. Thus, only the closed nodes will be tripped.

When all the tripping operations are successful, the fault isolation process ends. If any tripping operation fails, the corresponding node will be re-tripped for at most three times. If the node still refuses to trip, a correction tripping process will be carried out as follows:

Step 1: Change the type of node that has failed to trip from action node to monitoring node.

Step 2: Re-divide the monitoring regions and action regions, taking account of the change in Step 1.

Step 3: Re-locate the faulted region.

Step 4: Isolate the faulted region by following the process mentioned above.

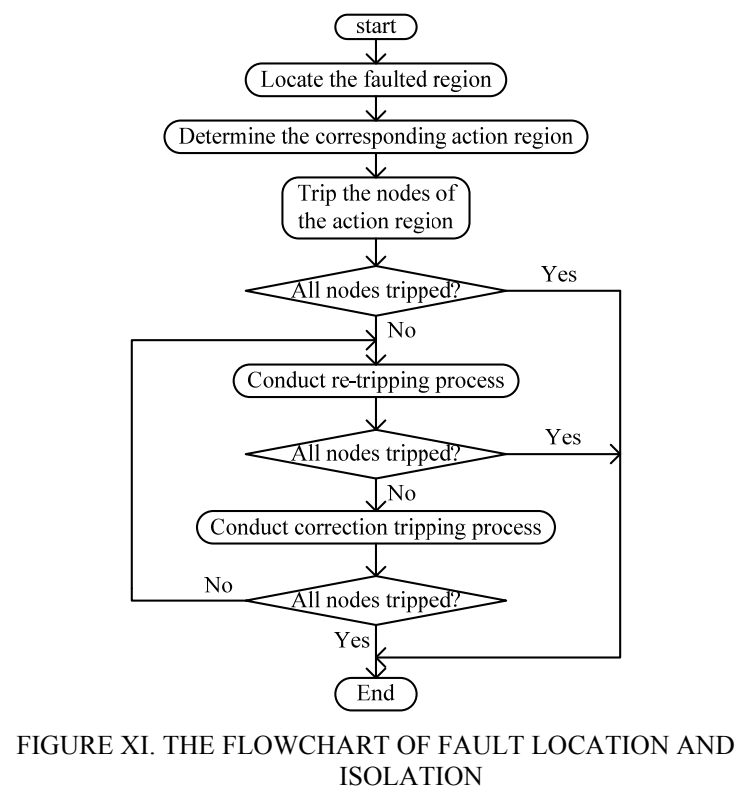

The flowchart of both fault location and isolation is shown in Figure XI.

In the example in Figure XII, the faulted region $\operatorname{Re}\left(\mathrm{B}_{3}, \mathrm{D}_{3}\right)$ is located correctly. Node $\mathrm{B}_{3}$ is not an action node. Its nearest action nodes $A_{3}$ and $A_{4}$, and the ending nodes $D_{3}$ and $D_{4}$ are put into $\boldsymbol{\Phi}=\left[\mathrm{A}_{3}, \mathrm{~A}_{4}, \mathrm{D}_{3}, \mathrm{D}_{4}\right]$. Consequently, the corresponding action region is $\operatorname{Re}\left(\mathrm{A}_{3}, \mathrm{~A}_{4}, \mathrm{D}_{3}, \mathrm{D}_{4}\right)$.

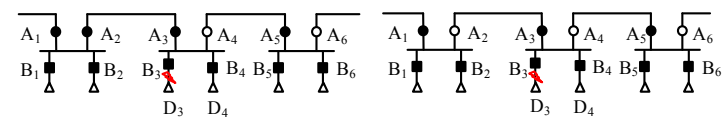

(A)

FIGURE XII. EXAMPLE OF FAULT ISOLATION. (A) THE TRIPPING OF $\mathrm{A}_{3}$ FAILS. (B) THE TRIPPING OF $\mathrm{A}_{2}$ SUCCEEDS

Node $A_{4}$ is a tie switch which is already open, only $A_{3}$ will be tripped. If the tripping succeeds, the fault isolation is completed. In this example both the tripping and the following re-tripping fail. During the correction tripping process $A_{2}$ is opened. The grid is now reconfigured in Figure XII(B).

\section{PROBLEM FORMULATION OF SERVICE RESTORATION}

The service restoration problem is a multi-objective and multi-constrained optimization problem [26]-[28]. Various objective functions and constraints are listed as follows, though the wording or selection of them may vary.

\section{A. Objectives}

The objectives, sometimes also called the indices, include

- Maximize Load Balance Degree

- Minimize load shedding

- Maximize the load restored with fewer switching operations 
Other objectives, for example minimization of the service restoration time and minimization of load losses, may be used for a superior solution.

Not all the objectives are used for a certain service restoration process.

1) Maximize load balance degree: The index to indicate how uniform the loads are distributed in a connected sub-grid, namely, Load Balance Degree, is defined as

$$
L B D=\frac{\min \left(l_{N . \%}\right)}{\max \left(l_{N . \%}\right)}
$$

where $\min \left(l_{N}, \%\right)$ and $\max \left(l_{N}, \%\right)$ are the minimum and maximum of the node load rates in a connected sub-grid.

Large LBD is preferable in practice. Therefore, one objective for service restoration is to obtain LBD as large as possible, i.e.

\section{$\max L B D$}

2) Minimize load shedding: That is

$$
\text { Min } \sum_{m \in \Omega} l_{R}(m)
$$

where $\Omega$ is the set of the load-shed regions.

3) Maximize the load restored with fewer switching operations: This is a comprehensive objective which can be formulated as

$$
\operatorname{Max} \frac{\sum_{m \in \mathrm{M}} \beta_{m} l_{R}(m)}{1+\alpha T}
$$

where $\boldsymbol{M}$ is the set of the regions to be restored, and $\beta_{m}$ is the parameter indicating the economic profit of the load in the $m$-th region. $T$ is the number of switches to be operated, which is weighted by a factor $\alpha$. In other words, this objective is to maximize the average economical profit for each switching operation.

\section{B. Constraints}

The possible electrical and operating constraints for service restoration include

- No load shedding

- No overload

- Current limits of nodes

- Grid topology

no loops among main source nodes

no more outage islands than the faulted action region

\section{- Controllability of nodes}

Like the objectives, not all the constraints are applied for a certain service restoration process.

The meanings of no load shedding and no overload are self-explanatory. Other constraints is explained as follows.

The current limit of a node is

$$
l_{N, \%}(i)<1.0
$$

It is the main source node that flows through the largest current, therefore this node is usually the only concern.

The topology constraint lies in two aspects, namely, no loops among main source nodes, which means to maintain the radiality of the grid, and no more outage islands than the faulted action region.

The controllability of the nodes is that some action nodes should not be operated.

Voltage limits are seldom adopted as a constraint for service restoration since the restoration solution is still a transitional plan [29].

\section{Search Space and Search Method}

The search space of service restoration solutions consists of discrete candidates. Each candidate is made up of a series of switching operations. It is an integer programming problem in combinatorial optimization to find a good solution in the search space. Tabu search, a metaheuristic algorithm, has been applied in a variety of areas yielding solutions whose quality is often better than that obtained by previous methods. Therefore, Tabu search has been used in our research to devise a close-tooptimal solution.

\section{Basic SCHEMES For SERVICE REStoration}

Four service restoration schemes are presented in this paper namely, real time service restoration, short time service restoration, service restoration with minimum load shedding, and modeled service restoration. The first two schemes are simple and basic ones, and will be discussed in this section. The other two advanced schemes will be described in part II.

\section{A. Real Time Service Restoration}

Real time service restoration is based on the region loads collected at the moment just before the fault.

The objective is to

- Maximize Load Balance Degree

- The constraints are

- No load shedding

- No overload

- Current limits of nodes

- Grid topology 


\section{- Controllability of nodes}

Because one of the constraints is no load shedding, therefore it makes sense that the number of the switching operation is not constrained.

Take the connected sub-grid in Figure XIII(A) for example. The rated current of all the nodes is $300 \mathrm{~A}$. The data in brackets are the region loads in A. The data without brackets are the loads of the main source nodes.

A permanent fault occurs in $\operatorname{Re}(B, C, N)$. By using the real time service restoration scheme, the service is successfully restored as shown in Figure XIII(B), with $L B D=0.87$.

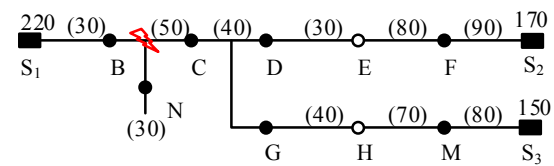

(A) A FAULT HAPPENS

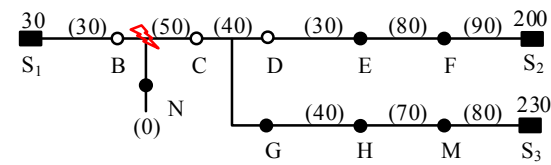

(B) THE SERVICE IS RESTORED

\section{FIGURE XIII. AN EXAMPLE OF THE REAL TIMERESTORATION}

\section{B. Short Time Service Restoration}

During the processing of a fault, there is a payback effect [30]. That is, the load has increased significantly when consumers, who were out of service, are being reenergized. This is called cold load pickup problems [31]-[33]. The scheme of the short time service restoration deals with these problems based on simple principles. It is different from the real time service restoration in that it restores the maximum loads forecasted for the near future, while the real time service restoration tries to restore the prefault loads. However, whether the scheme of short time service restoration is adopted depends on the availability of load forecast.

\section{CONCLUSIONS}

This is Part I of a two-part paper which presents systematical and fast approaches to FLISR on power distribution grids. Part I has introduced basic concepts such as node, region, and connected sub-grid. Nodes are classified into four types. A region is surrounded by several nodes of the same type. Regions are divided into two groups: the action region and the monitoring region. Nodes and regions are in hierarchies. A simplified load flow analysis for network reconfiguration is devised based on node-to-region and region-to-node load transformations within a connected sub-grid.

Five criteria can be used for fault location with sufficient fault information. The fault is first located in a monitoring region and then in an action region which is equal to or larger than the monitoring region. After that the action nodes of the action region are tripped to isolate the fault.

Four service restoration schemes have been proposed, and the first two of them have been introduce in Part I. Without load shedding, the real time service restoration transfers the load in the faulted region based on the prefault loads, whereas the short time service restoration is based on the loads forecasted for the near future.

The proposed approaches have been imbedded in 26 power distribution automation systems of $10 \mathrm{kV}$ grids since 2013 , and have been proven to be a great success.

\section{ACKNOWLEDGMENT}

Liang Ma received the B.Sc. Degree in electrical engineering from Changsha University of Science \& Technology, Hunan, China, in 2010. The same year he joined Xi' an Power Supply Company where he has been an assistant engineer since 2011. His main areas of interests include distribution network operation and maintenance.

Yi Xun received the B.Sc. Degree in electrical engineering from North China Electric Power University, Hebei, China, in 2010. The same year she joined Xi'an Power Supply Company where she has been an assistant engineer since 2011. Her main areas of interests include High-voltage electricity inspection and dedicated users' electricity bill settlement.

Zhihua Zhang received the B.Sc. and M.Sc. degrees in electrical engineering from Xi'an University of Science and Technology, Xi'an, Shaanxi Province, China, in 2009 and 2012 respectively. In 2000, he joined Shaanxi Electric Power Research Institute. His main research interests are in power distribution system analysis and protection, fault detection and location, and distributed generation.

Xiaoqing Zhang received the B.Sc. degree in electrical engineering from Xi'an University of Technology, Xi'an, Shaanxi Province, China, in 1993. The same year he joined Shaanxi Electric Power Research Institute where he has been a senior engineer since 2004. His main interests include power distribution system, protection, fault detection and location.

Di Yao received the B.Sc. degree in electrical engineering from Xi'an Polytechnic University, Xi'an, Shaanxi Province, China, in 2016. The same year she began to be a graduate student in her original school. Her main research interests are power distribution system analysis and protection.

\section{REFERENCES}

[1] J. Liu, X. Dong, X. Chen, X. Tong, X. Zhang, and S. Xu, Fault Location and Service Restoration for Electrical Distribution Systems. Singapore: Wiley, 2016, pp. 89-162.

[2] J. M. Solanki, S. Khushalani, and N. N. Schulz, "A multi-agent solution to distribution systems restoration," IEEE Trans. Power Syst., vol. 22, no. 3, pp. 1026-1034, Aug. 2007

[3] K. Jia, D. Thomas and M. Sumner, "a new single-ended fault-location scheme for utilization in an integrated power system," IEEE Trans. Power Del., vol. 28, no. 1, pp. 38-46, Jan. 2013.

[4] A. Borghetti, M. Bosetti, C. A. Nucci,M. Paolone, and A. Abur, "Integrated use of time-frequency wavelet decompositions for fault location in distribution networks: Theory and experimental validation," IEEE Trans. Power Del., vol. 25, no. 4, pp. 3139-3146, Oct. 2010.

[5] A. Borghetti, M. Bosetti, M. D. Silvestro, C. A. Nucci, and M. Paolone, "Continuous-wavelet transform for fault location in distribution power networks: definition of mother wavelets inferred from fault originated transients," IEEE Trans. Power Syst., vol. 23, no. 2, pp. 380-388, May 2008. 
[6] IEEE Guide for Determining Fault Location on AC Transmission and Distribution Lines, IEEE Standard C37.114, 2014.

[7] M. Majidi, A. Arabali and M. Etezadi-Amoli, "Fault location in distribution networks by compressive sensing," IEEE Trans. Power Del., vol. 30, no. 4, pp. 1761-1769, Aug. 2015.

[8] H. Nouri and M. M. Alamuti, "Comprehensive distribution network fault location using the distributed parameter model," IEEE Trans. Power Del., vol. 26, no. 4, pp. 2154-2162, Oct. 2011.

[9] V. V. Terzija, R. Ciric and H. Nouri, "Improved fault analysis method based on a new arc resistance formula," IEEE Trans. Power Del., vol. 26 no. 1, pp. 120-126, Jan. 2011

[10] M. M. Alamuti, H. Nouri, R. M. Ciric and V. Terzija, "Intermittent fault location in distribution feeders," IEEE Trans. Power Del., vol. 27, no. 1, pp. 96-103, Jan. 2012.

[11] Y. Liao, "Generalized fault-location methods for overhead electric distribution systems," IEEE Trans. Power Del., vol. 26, no. 1, pp. 53-64, Jan. 2011.

[12] K. Sun, Q. Chen, and Z. Gao, "An Automatic Faulted Line Section Location Method for Electric Power Distribution Systems Based on Multisource Information,” IEEE Trans. Power Del., vol. 31, no. 4, pp. 1542-1551, Aug. 2016.

[13] W. H. Chen, "Fault section estimation using fuzzy matrix-based reasoning methods," IEEE Trans. Power Del., vol. 26, no. 1, pp. 205213, Jan. 2011

[14] M. S. Choi, S. J. Lee, S. I. Lim, D. S. Lee and X. Yang, "A Direct ThreePhase Circuit Analysis-Based Fault Location for Line-to-Line Fault," IEEE Trans. Power Del., vol. 22, no. 4, pp. 2541-2547, Oct. 2007.

[15] F. C. L. Trindade, W. Freitas and J. C. Vieira, "Fault location in distribution systems based on smart feeder meters," IEEE Trans. Power Del., vol. 29, no. 1, pp. 251-260, Feb. 2014.

[16] M. Majidi, M. Etezadi-Amoli and M. Sami Fadali, "A novel method for single and simultaneous fault location in distribution networks," IEEE Trans. Power Syst., vol. 30, no. 6, pp. 3368-3376, Nov. 2015.

[17] S. Lotfifard, M. Kezunovic and M. J. Mousavi, "A systematic approach for ranking distribution systems fault location algorithms and eliminating false estimates," IEEE Trans. Power Del., vol. 28, no. 1, pp. 285-293, Jan. 2013.

[18] A. Shahsavari, S. M. Mazhari, A. Fereidunian and H. Lesani, "Fault indicator deployment in distribution systems considering available control and protection devices: a multi-objective formulation approach," IEEE Trans. Power Syst., vol. 29, no. 5, pp. 2359-2369, Sept. 2014.

[19] J. Ren, S. S. Venkata and E. Sortomme, "An accurate synchrophasor based fault location method for emerging distribution systems," IEEE Trans. Power Del., vol. 29, no. 1, pp. 297-298, Feb. 2014.

[20] G. A. Orcajo, J. M. Cano, M. G. Melero et al., "Diagnosis of electrical distribution network short circuits based on voltage Park's vector," IEEE Trans. Power Del., vol. 27, no. 4, pp. 1964-1972, Oct. 2012.

[21] S. Das, N. Karnik and S. Santoso, "Distribution fault-locating algorithms using current only," IEEE Trans. Power Del., vol. 27, no. 3, pp. 1144 1153 , July 2012

[22] J. H. Teng, W. H. Huang and S. W. Luan, "Automatic and fast faulted line-section location method for distribution systems based on fault indicators," IEEE Trans. Power Syst., vol. 29, no. 4, pp. 1653-1662, July 2014.

[23] N. Perera, A. D. Rajapakse and T. E. Buchholzer, "Isolation of faults in distribution networks with distributed generators," IEEE Trans. Power Del., vol. 23, no. 4, pp. 2347-2355, Oct. 2008.

[24] J. Huang and K. A. Corzine, "AC impedance measurement by line-toline injected current," in Proc. IEEE Ind. Appl. Conf., 2006, pp. 300306.

[25] X. Long, W. Xu and Y. W. Li, "A new technique to detect faults in deenergized distribution feeders-part I: scheme and asymmetrical fault detection," IEEE Trans. Power Del., vol. 26, no. 3, pp. 1893-1901, July 2011.

[26] N. D. R. Sarma, S. Ghosh, K. S. P. Rao, and M. Srinivas, "Real time service restoration in distribution networks," IEEE Trans. Power Del., vol. 9, no. 4, pp. 2064-2070, Oct. 1994
[27] K. N. Miu, H.-D. Chiang, B. B. Yuan, and G. Darling, "Fast service restoration for large-scale distribution systems with priority customers and constraints," IEEE Trans. Power Syst., vol. 13, no. 3, pp. 789-795, Aug. 1997.

[28] Y. Kumar, B. Das, and J. Sharma, "Multiobjective, multiconstraint service restoration of electric power distribution system with priority customers,” IEEE Trans. Power Del., vol. 23, no. 1, pp. 261-270, Jan. 2008.

[29] D. Shirmohammadi, "Service restoration in distribution networks via network reconfiguration," IEEE Trans. Power Del., vol. 7, no. 2, pp. 952-958, Apr. 1992

[30] D. S. Popovic and Z. N. Popovic, "A risk management procedure for supply restoration in distribution networks," IEEE Trans. Power Syst., vol. 19, no. 1, pp. 221-229, Feb. 2004.

[31] C. Ucak and A. Pahwa, "An analytical approach for step-by-step restoration of distribution systems following extended outages," IEEE Trans. Power Del., vol. 9, no. 3, pp. 1717-1723, Jul. 1994.

[32] V. Gupta and A. Pahwa, "A voltage drop-based approach to include cold load pickup in design of distribution systems," IEEE Trans. Power Syst., vol. 19, no. 2, pp. 957-963, May 2004.

[33] V. Kumar, R. H. C. Kumar, I. Gupta, and H. O. Gupta, "DG integrated approach for service restoration under cold load pickup," IEEE Trans. Power Del., vol. 25, no. 1, pp. 398-406, Jan. 2010. 